\title{
Material-Driven Fibronectin Assembly Promotes Maintenance of Mesenchymal Stem Cell Phenotypes
}

\author{
Patricia Rico,* Hayk Mnatsakanyan, Matthew J. Dalby, and Manuel Salmerón-Sánchez*
}

Mesenchymal stem cells (MSCs) are a research tool to investigate fundamental biology and are candidates for use in regenerative medicine. In this context, significant efforts have been devoted to develop technologies to control stem cell fate, including the use of soluble factors in media. However, material properties offer alternative approaches that avoid the use of soluble factors. Here, a material system capable of sustaining the growth of stem cells (maintaining stemness) and of promoting highly efficient differentiation upon external stimulation is described. Poly(ethyl acrylate) induces assembly of fibronectin (FN) into nanonetworks (FN fibrillogenesis) upon simple adsorption from solutions. It is shown that these FN nanonetworks allow growth of MSCs and maintenance of stemness for long periods of time (up to $30 \mathrm{~d})$ using basal media in absence of soluble factors. Additionally, the system promotes enhanced levels of differentiation when defined supplemented media are used. The study reveals the critical role of the intermediate protein layer at the material interface to control MSC fate regardless of the properties of the underlying material and it introduces a new material system as a candidate to be used in MSC niche design.

\section{Introduction}

Mesenchymal stem cells (MSCs) are capable of self-renewal and multilineage differentiation-classically into osteoblasts, adipocytes, chondrocytes, and fibroblasts. ${ }^{[1,2]}$ Multipotent cells remain in their niche as slow proliferating, metabolically quiescent cells

Dr. P. Rico

Biomedical Research Networking Center in

Bioengineering, Biomaterials and

Nanomedicine (CIBER-BBN)

Camino de Vera s/n, 46022 Valencia, Spain

E-mail:parico@upvnet.upv.es

Dr. P. Rico, H. Mnatsakanyan

Center for Biomaterials and Tissue Engineering (CBIT)

Universitat Politècnica de València

Camino de Vera s/n, 46022 Valencia, Spain

Prof. M. I. Dalby

Center for Cell Engineering, Institute of Molecular

Cell and Systems Biology

CMVLS

University of Glasgow

Joseph Black Building, Glasgow G12 8QQ, UK

Prof. M. Salmerón-Sánchez

Division of Biomedical Engineering

School of Engineering

University of Glasgow

Rankine Building, Glasgow G12 8LT, UK

E-mail: Manuel.Salmeron-Sanchez@glasgow.ac.uk

DOI: 10.1002/adfm.201602333 in order to maintain multipotency. When using MSCs for regenerative medicine, it is important to obtain a sufficient number of cells that maintain pluripotency without compromising MSC senescence..$^{[3,4]}$

Material systems that mimic the natural niche environment of MSCs may offer an alternative to the use of complex cocktails of soluble factors used in the culture media. Previous studies show that the cell/material interface plays an essential role on MSC function and differentiation, encompassing promising approaches to manipulate differentiation of stem cells ranging from chemistry, ${ }^{[5-7]}$ surface modifications, ${ }^{[8,9]}$ topography, ${ }^{[10-12]}$ stiffness, ${ }^{[13,14]}$ and even dynamic material properties such as stress relaxation. ${ }^{[15]}$

While we are starting to identify the range of materials properties that can be used to drive stem cell differentiation, there is much left to discover and understand. In addition, cells do not feel the surface of materials directly, but through an intermediate layer of adsorbed proteins. The conformation and distribution of this protein layer will determine integrin binding and the organization of focal adhesions, which in turn will influence cell signaling and hence fate. ${ }^{[16-20]}$

Acrylates are common biomaterials with tunable physical properties. ${ }^{[21]}$ In this work we used substrates that slightly differ in surface chemistry, varying only one methyl group in the side chain-poly(ethyl acrylate) (PEA) and poly(methyl acrylate) (PMA). Using this material system, we have previously demonstrated that this subtle variation in surface chemistry modulates the conformation of adsorbed fibronectin (FN). Typically, FN adsorbs to synthetic materials in a globular morphology, as it does on PMA. However, on PEA, the FN molecules spontaneously organize into nanonetworks, a process that we have termed material-driven fibronectin fibrillogenesis. ${ }^{[22-24]}$ We hypothesize that these FN nanonetworks assembled on PEA influence the behavior of MSCs. In this new report, we have investigated the role of FN nanonetworks on MSC adhesion, differentiation (osteogenic, adipogenic), and growth, by culturing cells in absence of differentiation factors.

\section{Results}

We have used C3H10T1/2 cells, an established murine multipotent mesenchymal stem (mMSC) cell line from 14-to 17-d-old 


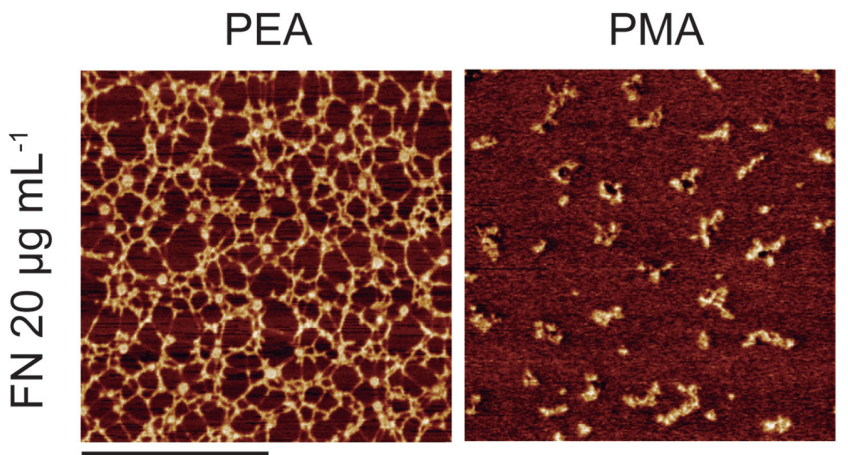

Figure 1. FN conformation on material substrates. PEA and PMA coated from a solution of $20 \mu \mathrm{g} \mathrm{mL}^{-1} \mathrm{FN}$. Images obtained by AFM. Scale bar is $0.5 \mu \mathrm{m}$.

C3H whole mouse embryo. ${ }^{[25]}$ These cells display fibroblastic morphology in high-density cell cultures and are functionally similar to mesenchymal stem cells. They have the potential to differentiate into mesodermal lineages (reticular, adipogenic, osteogenic, and chondrogenic) under defined conditions, maintain a stable morphology in culture, and present no tumorigenic activity. ${ }^{[26]}$

\subsection{FN Adsorption}

Samples of PEA and PMA consisting of a vinyl backbone chain with side group $-\mathrm{COO}(\mathrm{CH} 2)_{x} \mathrm{H}$, with $x=1$ for PMA and $x=2$ for PEA were coated with $20 \mu \mathrm{g} \mathrm{mL}^{-1} \mathrm{FN}$. We had previously described the molecular distribution of $\mathrm{FN}$ on the different substrates by atomic force microscopy (AFM). ${ }^{[16]}$ Figure 1 shows FN organized into nanonetworks upon adsorption on PEA and globular FN aggregates on PMA. The surface density of adsorbed FN was previously determined by western blot analysis and the results showed similar values for both PEA and PMA, resulting in $\approx 340 \mathrm{ng} \mathrm{cm}^{-2}$. [22]

\subsection{Cell Adhesion}

We first examined the effect of FN organization (fibrillar on PEA vs globular on PMA) on mMSC adhesion after $3 \mathrm{~h}$ of culture. It is important to highlight that cell adhesion was performed in the absence of serum in the media (only Dulbecco's modified Eagle medium (DMEM) supplemented with antibiotics), thus initial cell/material contact occurs only via interaction with adsorbed FN on the material substrates. Cells were seeded at a low density $\left(5000\right.$ cells $\left.\mathrm{cm}^{-2}\right)$ to maximize cell-material interactions and to minimize cell-cell contacts. Figure 2a shows cell morphology after staining actin fibers

a)

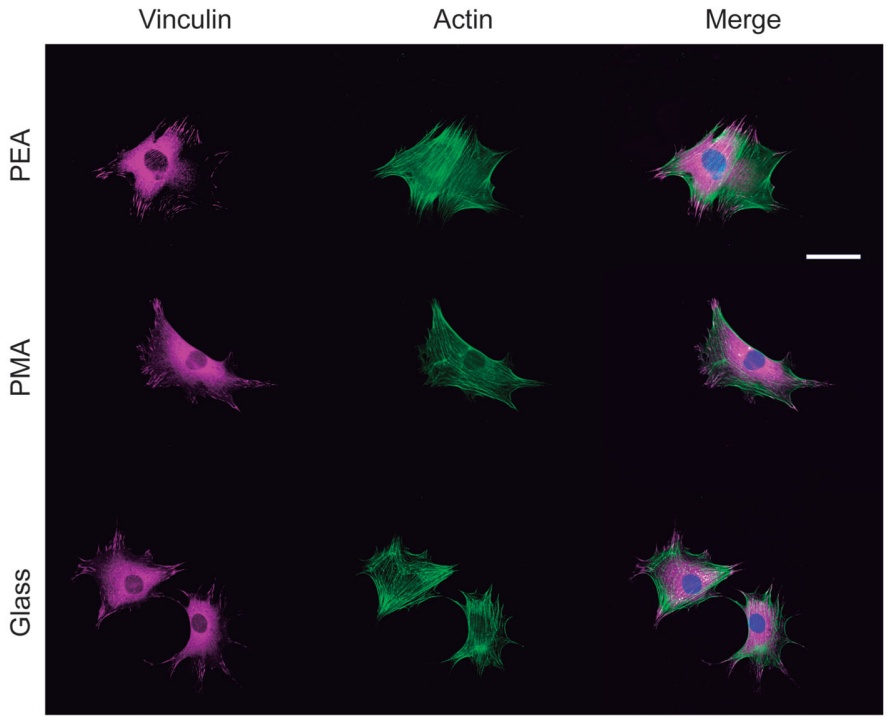

b)

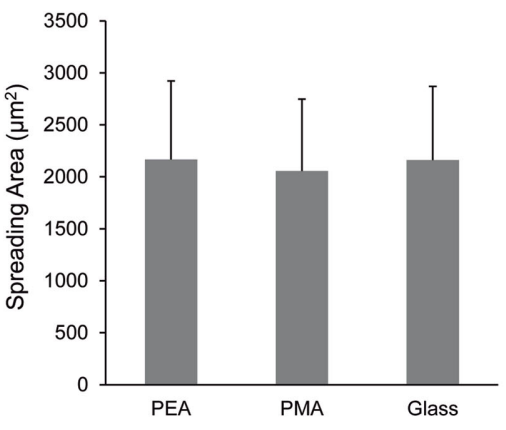

and vinculin within focal adhesion complexes (other focal adhesion proteins, e.g., tensin and talin, are shown in Figure S1, Supporting Information). Cells displayed prominent actin stress fibers terminating at well-developed focal adhesion sites. Cell area presented similar values for all surfaces (Figure 2b) although circularity of cells was slightly higher on PMA than PEA (Figure 2c).

Focal adhesion size distribution was quantified by image analysis on the different substrates (Figure 3a,b). We found significant differences for the total area occupied by focal adhesions (Figure 3a): PEA > PMA > control glass. The frequency distribution was similar on all surfaces, with a higher fraction of smaller plaques ( 1 or $2 \mu \mathrm{m}^{2}$ ) that decreased monotonically up to larger, $4 \mu^{2}$, plaques (Figure $3 b$ ). Immunofluorescence staining for other focal adhesion proteins, e.g., talin and tensin, revealed similar trends (Figure S1, Supporting Information).

To gain insights into the initial signaling mechanism, we examined activation (phosphorylation) of focal adhesion kinase (FAK) after $3 \mathrm{~h}$ of culture in absence of serum in medium. FAK localizes to focal adhesions and forms a main part of the adhesion signaling strata. Figure $3 c$ shows expression of pFAK/FAK. 


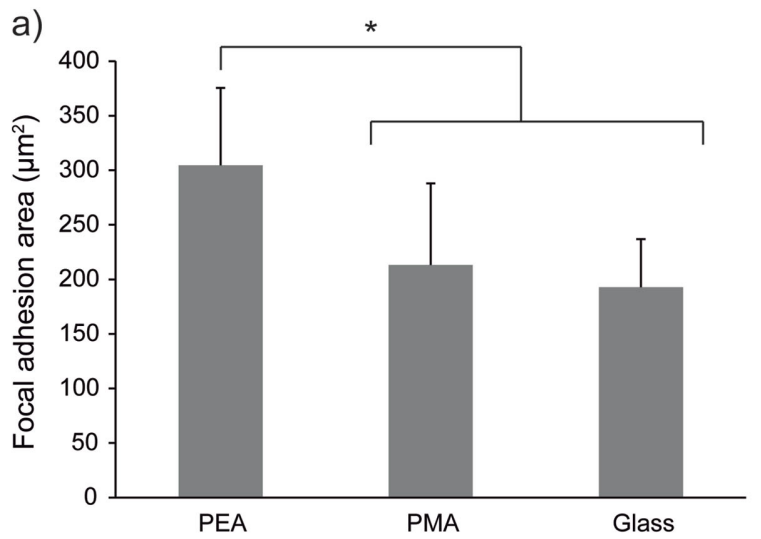

b)

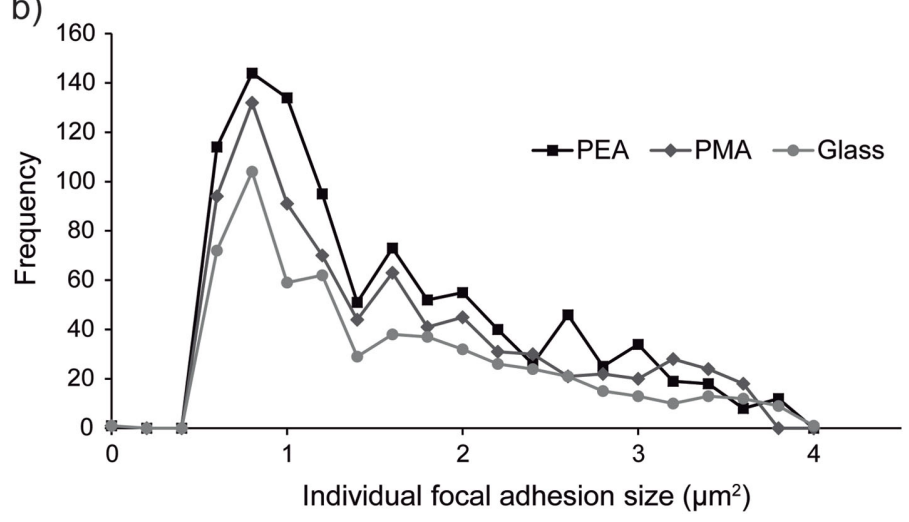

c)
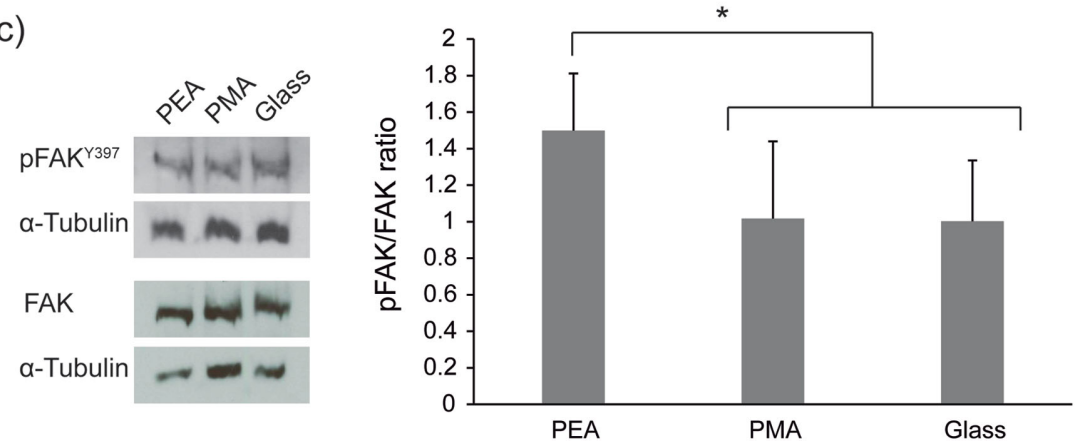

Figure 3. Focal adhesion and cell signaling. a) Quantification of focal adhesion area on cells cultured on different substrates. PEA showed the highest values followed by PMA and Glass. b) Frequency distribution for focal adhesion size. PEA showed the higher number of individual focal adhesions, the trend was similar for all substrates. c) Western blot for pFAK and FAK from cells grown onto different substrates. Normalized pFAK/FAK ratio showed high levels for PEA compared to PMA and Glass substrates. a) ( $n=10$ cells) and c) Three independent experiments represented as mean \pm standard deviation. $* p<0.05$.

Higher levels of pFAK were obtained on PEA compared with PMA and glass, revealing increased FAK activation from the FN nanonetworks assembled on PEA fitting nicely with the observation of increased adhesion area.

\subsection{Cell Differentiation}

We first evaluated the effect of FN assembly (globular on PMA vs nanonetworks on PEA) on mMSC differentiation using basal medium (B.M.) containing 10\% fetal bovine serum (FBS) without any supplements or growth factors in it. Considering the range of stiffness of PEA and PMA used in our experiments $(\approx 1000-2000 \mathrm{kPa}),{ }^{[27]}$ and the range of stiffness that cells are sensitive to $(\approx 1-40 \mathrm{kPa}),{ }^{[13]}$ cells are unable to deform the underlying substrates that will be sensed simply as rigid substrates. We evaluated the phenotypical behavior of mMSCs during commitment along two different lineages: osteogenic and adipogenic, and also their ability to grow maintaining stemness.

We used cell seeding densities of 10000 cells $\mathrm{cm}^{-2}$ to promote osteogenesis and 30000 cells $\mathrm{cm}^{-2}$ to favor adipogenesis; cells were seeded at a density of 10000 cells $\mathrm{cm}^{-2}$ in basal medium for basal conditions. ${ }^{[28]}$ Immunofluorescence images were taken after $15 \mathrm{~d}$ of culture in order to analyze morphology of cells onto FN-coated materials (PEA and PMA were subjected to basal conditions, B.M., and glass was used as positive control with differentiation medium, D.M.). Figure 4 shows that mMSCs grew to confluence and consistently displayed a spindle-like, fibroblastic morphology across FN-coated PEA and PMA surfaces, which suggests no spontaneous differentiation. ${ }^{\text {28,29] }}$ Cells on glass substrates (control using D.M.) presented a polygonal morphology typical of osteoblasts after an osteogenic induction period (osteogenic differentiation medium, O.D.M.) and a rounded shape due to the high content of vacuoles characteristic of adipocytes after adipogenic induction (adipogenic differentiation medium, A.D.M.). ${ }^{[30]}$

Further, we investigated specific markers of osteogenic differentiation (osteopontin (OPN) and Runt related transcription factor 2 (Runx2)) as well as Oil red O staining to assess adipogenic commitment in identical basal conditions for PEA and PMA (B.M.) and differentiation conditions (O.D.M. and A.D.M.) for glass controls. Immunofluorescence was done after 15 days of culture for OPN, while Runx2, an early expression transcription factor in osteogenenesis, was detected after $3 \mathrm{~d}$ of culture. ${ }^{[31]}$ Oil Red O staining confirmed the presence of adipocytes after $15 \mathrm{~d}$ of culture. Figure 5 shows representative images on the different substrates and the corresponding quantification via image analysis of staining intensity. Both mMSCs on FN-coated PEA and PMA (using B.M.) showed minimum levels of OPN compared with glass (using D.M., positive control of differentiation). In contrast Runx2 levels were similar between PMA and PEA, whereas Runx2 levels were slightly higher on glass. Note that Runx2 was always evaluated after $3 \mathrm{~d}$ of osteogenic stimulation, as it is a transcription factor that is expressed early 

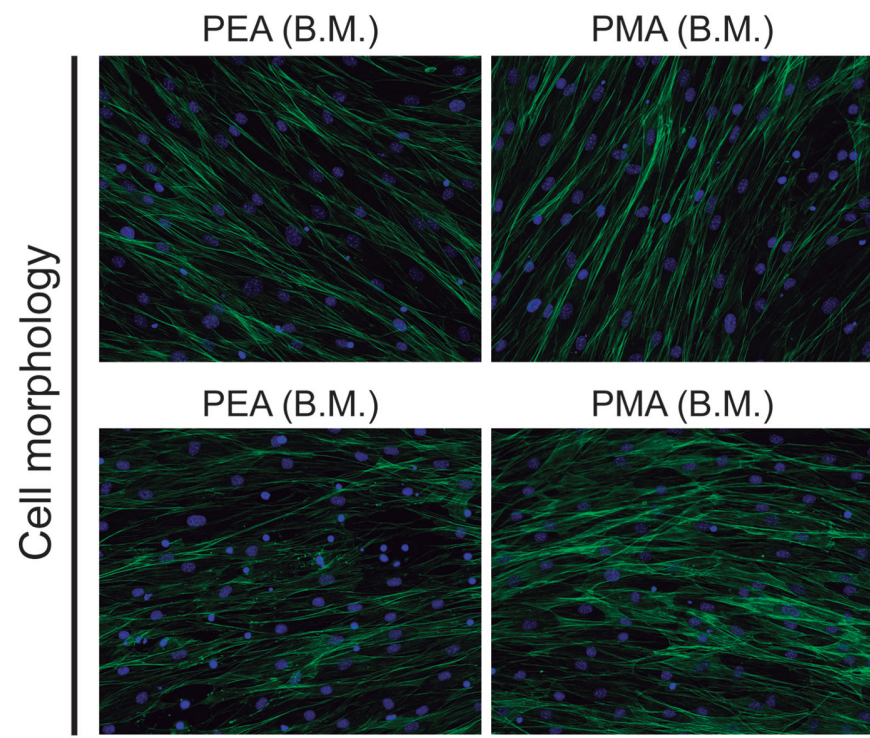

Glass (+ O.D.M.)

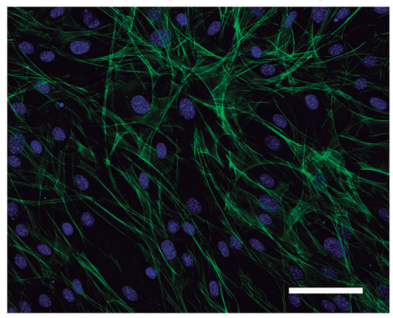

PMA (B.M.)

Glass (+ A.D.M.)
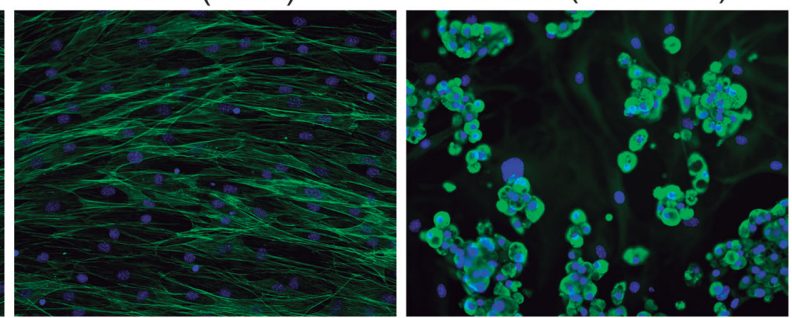

Figure 4. mMSCs phenotypes. Fluorescence staining showing actin cytoskeleton (green) and nuclei (blue) for evaluation of morphological features of mMSC. Cells cultured onto FN-coated PEA and PMA substrates under basal media conditions (B.M.) during 15 d. No spontaneous differentiation was observed. Control glass was cultured under osteogenic (osteogenic differentiation medium, +O.D.M.) and adipogenic stimulating conditions (adipogenic differentiation medium, +A.D.M.). Cell morphology showed characteristic morphologies of osteoblast and adipocytes, respectively. Scale bar $50 \mu \mathrm{m}$.

in the osteogenic cascade to activate genes involved in osteoblast differentiation. In respect to adipogenic markers, Oil red O staining only revealed lipid vacuoles that had developed fat droplets (Figure 5, inset magnification) on the positive control of differentiation (glass + A.D.M.). We also analyzed other osteogenic markers such as osteocalcin and integrin binding sialoprotein with similar results (PEA and PMA showing minimum levels of staining, Figure S2, Supporting Information).

Based on these results, we hypothesized that mMSCs cultured on FN-coated substrates maintained their self-renewal potential rather than differentiating into specific lineages. Figure 5 shows high levels of Sca1 staining for PEA and PMA, a specific marker of undifferentiated murine mesenchymal cells. ${ }^{[32,33]}$

These results suggest that FN-coated PEA and PMA promote maintenance of stemness rather than lineage commitment in the absence of soluble factors in media. Next, we evaluated their ability to sustain MSCs differentiation under defined media conditions and also the self-renewal maintenance in longer-term cultures. To do this, we designed three different experimental conditions: (i) mMSCs cultured for $15 \mathrm{~d}$ using differentiation media (to evaluate the potential of $\mathrm{FN}$ coated PEA and PMA to support osteogenic/adipogenic commitment); (ii) mMSCs cultured for $15 \mathrm{~d}$ under basal conditions and then stimulated to differentiate with differentiation media for another $15 \mathrm{~d}$; and (iii) mMSCs cultured for $30 \mathrm{~d}$ under basal conditions.

Figure 6 shows representative images of different experimental conditions and their quantification using image analysis. Runx2 and OPN expression was detected at similar levels in cells on PEA, PMA, and control glass after 3 (Runx2) and $15 \mathrm{~d}(\mathrm{OPN})$ of stimulation with osteogenic medium (O.D.M.). Likewise, Oil red O staining showed formation of lipid vacuoles containing fat droplets in all substrates after $15 \mathrm{~d}$ of stimulation with adipogenic medium (A.D.M.). These results show the ability of FN-coated substrates (PEA and PMA) to support efficient lineage commitment under differentiation conditions. However, when mMSCs were cultured for $15 \mathrm{~d}$ under basal conditions (B.M.) and afterward stimulated with osteogenic/ adipogenic media, (note that cells were stimulated with differentiation media for $15 \mathrm{~d}$ in all cases but for Runx 2 where still differentiation stimuli were maintained for $3 \mathrm{~d}$ ) the capacity of FN-coated substrates to promote lineage commitment was particularly strong for cells cultured on FN nanonetworks on PEA, as revealed by the higher levels of osteoblastic differentiation (Figure 6).

Furthermore, after 15 and $30 \mathrm{~d}$ of culture in basal medium (15 d in B.M./30 d in B.M.), PEA presented again higher levels of stemness markers (Sca 1) compared to PMA and glass that showed minimal levels of expression (Figure 6).

\subsection{Analysis of Gene Expression}

After this initial evaluation of results obtained for cell differentiation on the FN-coated substrates, we performed real time quantitative polymerase chain reaction (qPCR) analysis of specific genes related to osteogenic or adipogenic commitment under basal conditions (B.M.). Runx2 and adipocyte peroxisome proliferation-activated receptor (PPAR $\gamma 2$ ) are transcription factors involved in osteogenic and adipogenic commitment respectively. As both transcripts/proteins are involved in early onset of lineage commitment, we first analyzed their expression after $3 \mathrm{~d}$ of culture in basal medium (B.M.): Figure 7a(i) shows that Runx2 levels were higher in PEA while in PMA and control glass remains similar; in contrast, PPAR $\gamma 2$ levels were low both in PEA and PMA. Further qPCR analysis was performed after $3 / 15 \mathrm{~d}$ of culture in differentiation media prior to 

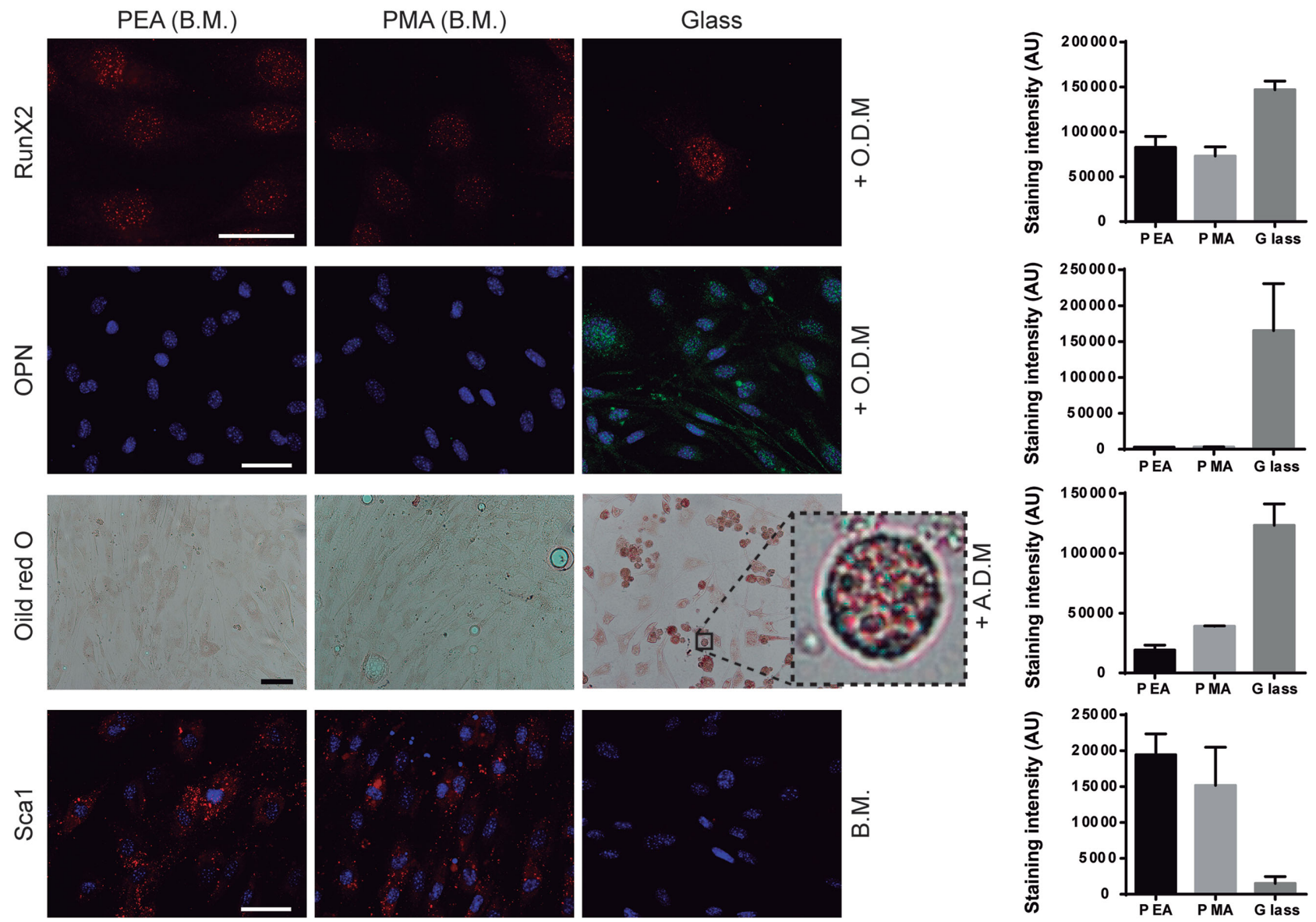

Figure 5. mMSCs under basal conditions. Immunofluorescence for osteogenic, adipogenic, and stemness markers expressed under basal conditions and quantification of staining intensity. Osteogenic markers: Runx2 (red), OPN (green); Sca 1 was used as stemness marker (red); Nuclei are showed in blue; adipogenic commitment was evaluated by Oil red O staining. Control substrates were cultured under differentiation medium (+O.D.M.) and adipogenic differentiation medium (+A.D.M.). PEA and PMA showed minimum levels of OPN and Oil red O staining under basal conditions (B.M.). Runx2 expression was higher on PEA. Stemness marker (Sca1) expression was elevated only in FN-coated PEA and PMA substrates under basal conditions (B.M.). Scale bar $50 \mu \mathrm{m}$.

maintaining cells for $15 \mathrm{~d}$ in basal medium (B.M.): Figure 7a(ii) represents Runx2 and OPN as markers of osteogenesis whereas Figure $7 \mathrm{a}$ (iii) represents relative expression of PPAR $\gamma 2$ and lipoprotein lipase (LPL) as markers of adipogenesis. Expression levels of PPAR $\gamma 2$ and LPL were minimal in all substrates compared to the positive control for differentiation. OPN levels were also low and Runx 2 levels were lower than that observed at $3 \mathrm{~d}$; this is in agreement with previous immunofluorescence data (Figure 5). We also analyzed relative expression of stemness markers Sca 1 and CD29 under basal conditions (iv). PEA presented the highest levels of Sca 1 and CD29 followed by PMA and then minimum expression levels for these markers was noted for the positive differentiation controls (Figure 7a(iv)). These results are consistent with data obtained in immunofluorescence images (Figures 5 and 6).

We next used qPCR analysis to study expression of the osteogenic, adipogenic, and stem cell-related genes for cells on the FN-coated substrates cultured for $15 \mathrm{~d}$ (Figure $7 \mathrm{~b}$ ) with (1) mMSCs under differentiation conditions (Figure 7b(i)); (2) mMSCs cultured for $15 \mathrm{~d}$ with basal media and then stimulated to commitment with differentiation media (Figure 7b(ii)); and finally evaluation of stemness after a $30 \mathrm{~d}$ of long-term culture in B.M. (Figure 7b(iii)).

FN-coated PEA and PMA substrates showed a similar potential to support induction of both osteogenic and adipogenic differentiation under defined media conditions compared to inducement on glass control (Figure 7b(i)). When mMSCs were cultured for $15 \mathrm{~d}$ prior to addition of defined medias, a trend of increased induction on PEA was noted (Figure $7 \mathrm{~b}(\mathrm{ii})$ ) in agreement with results obtained at protein level (Figure 6). Note that only on PEA, cells maintain their ability to differentiate into osteogenic and adipogenic lineages, whereas on PMA only the ability to undergo adipogenesis is maintained after $15 \mathrm{~d}$ of culture in B.M. ((Figure $7 \mathrm{~b}(\mathrm{ii}))$. Further note that we have applied appropriate statistics and reported differences based on $p$ values. However, we acknowledge that these variations even if consistent at the gene and proteins expression are small.

Importantly, high levels of stemness markers were only found in PEA after $30 \mathrm{~d}$ of culture (Figure $7 \mathrm{~b}$ (iii)), in line with data previously obtained by immunofluorescence assays (Figure 6). 
RunX2

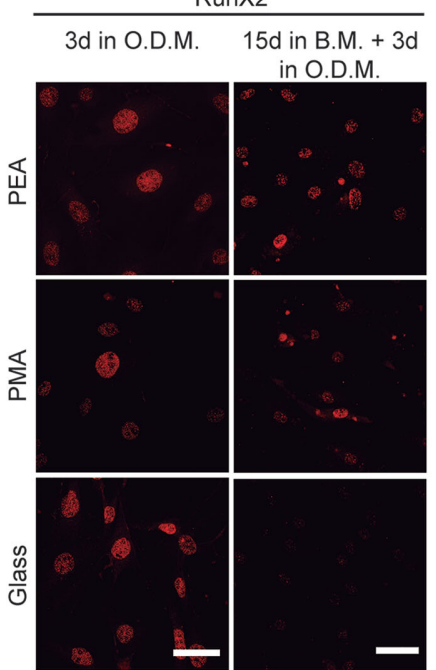

Oil red $\mathrm{O}$

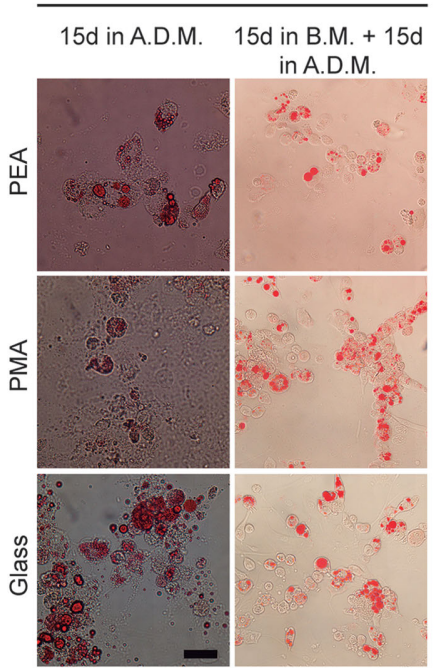

3d in O.D.M.

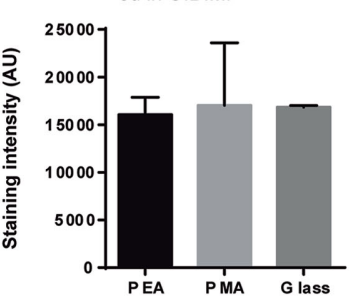

$15 d$ in B.M. $+3 d$ in O.D.M

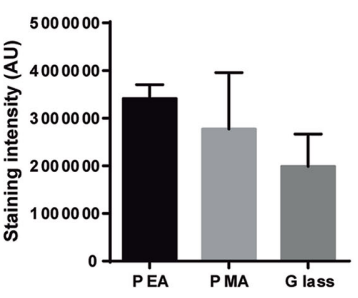

$15 d$ in A.D.M.

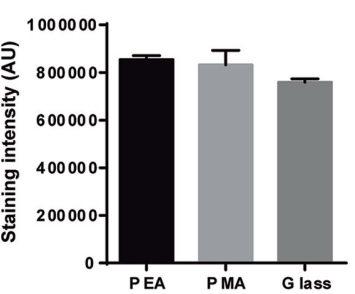

$15 d$ in B.M. $+15 d$ in A.D.M.

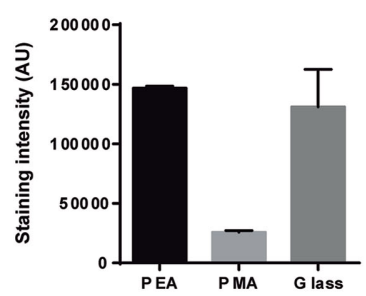

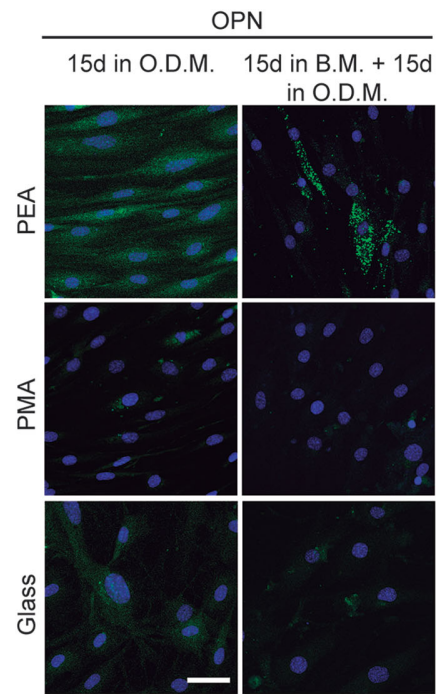
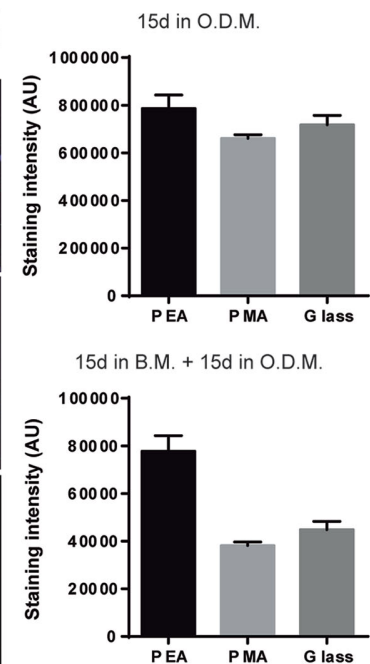

$15 d$ in B.M.

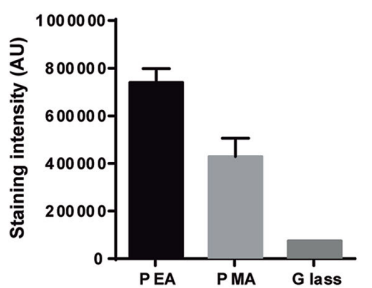

$30 \mathrm{~d}$ in B.M.

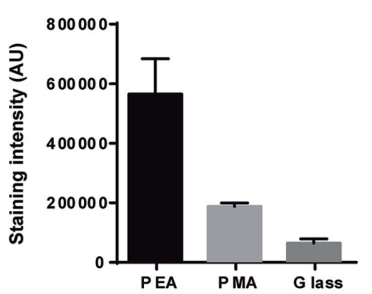

Figure 6. mMSCs under differentiation conditions. Immunofluorescence for osteogenic, adipogenic, and stemness markers expressed under differentiation conditions and quantification of staining intensity. Immunofluorescence for osteogenic differentiation: Runx2 (red), OPN (green); Sca1 was used as stemness marker (green); nuclei are shown in blue; adipogenic commitment by Oil red O staining. FN-coated PEA and PMA showed similar potential to induce osteogenic/adipogenic phenotypes under differentiation conditions (osteogenic, +O.D.M. and adipogenic, +A.D.M.) during 15 d of culture compared to glass differentiated control. When cell cultures were performed with a previous treatment of $15 \mathrm{~d}$ in basal media (B.M.) and subsequently induced to differentiate, FN-coated PEA showed the high levels in osteogenic markers as well as stemness maintenance after $30 \mathrm{~d}$ of culture.

\subsection{Analysis of Contractility}

Forces generated by cells regulate different processes including cell contraction and differentiation, ${ }^{[34]}$ and cell migration ${ }^{[35]}$ via phosphorylation of the myosin light chain (MLC). ${ }^{[36]}$ We examined whether this contractility process was related to differentiation or self-renewal of mMSCs cultured on PEA and PMA. Immunofluorescence staining for phosphorylated MLC (pMLC) revealed colocalization of pMLC coincident with the actin cytoskeleton in cells cultured on all substrates (Figure S3, Supporting Information). Cells were then cultured in the presence of pharmacological inhibitors that impair contractility. We used Y-27632 as specific inhibitor of Rho-kinase, and blebbistatin as specific inhibitor of myosin II activity. ${ }^{[37,38]}$ Staining for pMLC was reduced in a dose-dependent manner $\left(10 \times 10^{-6}\right.$ and $20 \times 10^{-6} \mathrm{M}$, respectively) in the presence of these inhibitors in cells on the different substrates (Figure S3, Supporting Information).

The expression of osteogenic markers requires high cell contractility, ${ }^{[5,6]}$ as expected, inhibition of cell contractility (blebbistatin and Y-27632 at final concentrations of $20 \times 10^{-6} \mathrm{M}$ ) greatly reduced osteogenic marker expression from the MSCs on all substrates (Figure 8).

Self-renewal markers were also studied and CD29 and Sca1 expression levels were strongly reduced regardless of the FN distribution on PEA and PMA (Figure 8a,b), which suggests a role of material-activated cell contractility in stemness maintenance. The motivation of the contractility experiments was to assess if maintenance of phenotype on the polymers (on PEA in particular) was related to cytoskeletal tension and fitted in with the MSC tension-phenotype relationship, e.g., low MSC 
a)

b)

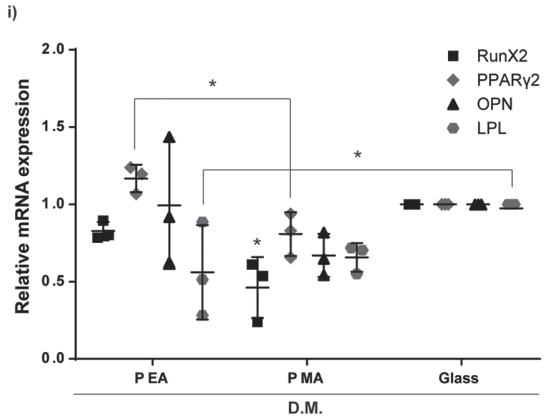

iii)
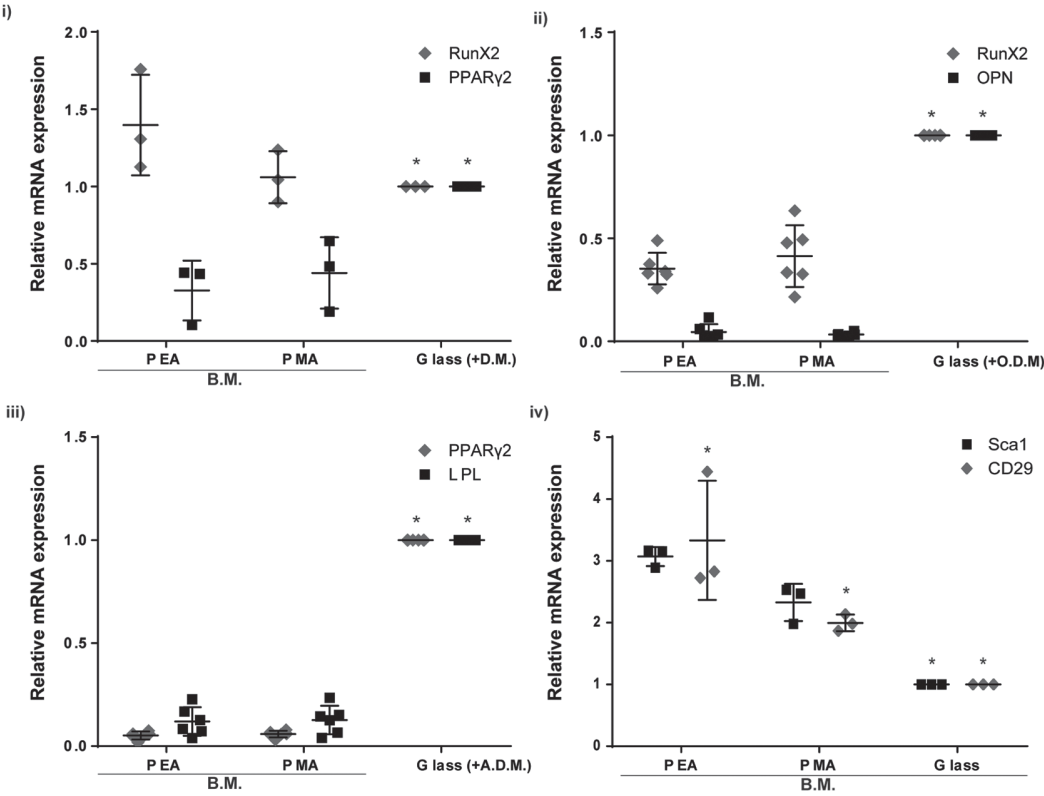

ii)

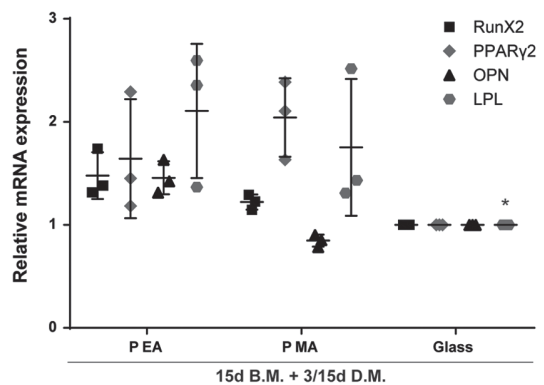

iii)

Figure 7. Analysis of relative mRNA expression under basal and differentiation conditions. a) mRNA expression under basal conditions. i) Cells cultured under basal conditions (B.M.). Representation of relative levels of mRNAs of early transcription factors that determine lineage commitment after $3 \mathrm{~d}$ of culture: Runx2 for osteogenic commitment and PPAR $\gamma 2$ for adipogenic commitment. ii,iii) Cells cultured $15 \mathrm{~d}$ under basal conditions (B.M.). Control glass was induced to differentiate under osteogenic (+O.D.M.) and adipogenic medium (+A.D.M.). Representation of relative levels of mRNAs of genes markers of osteogenesis (Runx2 and OPN) and adipogenesis (PPAR $\gamma 2$ and LPL). iv) Cells cultured $15 \mathrm{~d}$ under basal conditions (B.M.). Representation of relative levels of mRNAs of genes markers of stemness maintenance (Sca1 and CD29) after $15 \mathrm{~d}$ of culture. b) mRNA expression under differentiation conditions. i) Cells cultured under differentiation conditions (osteogenic, +O.D.M. and adipogenic medium, +A.D.M.). Representation of relative levels of mRNAs of genes markers of osteogenesis (Runx2 and OPN) and adipogenesis (PPAR $\gamma 2$ and LPL) after $3 \mathrm{~d}$ (Runx2 and PPAR $\gamma 2$ ) and $15 \mathrm{~d}$ of culture (OPN and LPL). ii) Cells previously cultured under basal conditions for $15 \mathrm{~d}$, and subsequently stimulate to differentiate cultured for $15 \mathrm{~d}$ with osteogenic (+O.D.M.) or adipogenic (+A.D.M.) medium. Representation of relative levels of mRNAs of genes markers of osteogenesis (Runx2 and OPN) and adipogenesis (PPAR $\gamma 2$ and LPL) after $3 \mathrm{~d}$ (Runx2 and PPAR $\gamma 2$ ) and $15 \mathrm{~d}$ of culture (OPN and LPL). iii) Cells cultured under basal conditions for $30 \mathrm{~d}$. Representation of relative levels of mRNAs of genes markers of markers of stemness maintenance (Sca1 and CD29). FN-coated PEA showed high expression of stemness markers.

intracellular tension causing adipogenesis and high MSC intracellular tension driving osteogenesis. ${ }^{[6]}$

\section{Discussion}

The application of materials science to modulate the cellular microenvironment and direct MSC fate will be fundamental to developing an understanding of stem cell function. Different attempts include cues such as topography ${ }^{[39]}$ and stiffness ${ }^{[13]}$ or chemistry. ${ }^{[40]}$ While these studies demonstrate that material properties influence cell fate decisions, no examples so far have observed material-induced conditions that permit first MSC growth and then enhanced differentiation. ${ }^{[12,14]}$ Here we show that FN-nanonetworks assembled on PEA promote enhanced, prolonged maintenance of self-renewal and retention of functional multipotency when basal media is used. However, when defined medias, traditionally used to induce specific differentiations, enhanced levels of differentiation is noted; i.e., the cells were more sensitive to the defined medias when cultured on the FN nanonetworks on PEA.

It is well documented that cell-material interaction takes place through an intermediate layer of proteins adsorbed onto substrates. The amount of protein adsorbed and its conformation on materials depends of surface chemistry. ${ }^{[40]}$ Proper conformation of adsorbed proteins directs integrin binding, 
a)

Y-27632

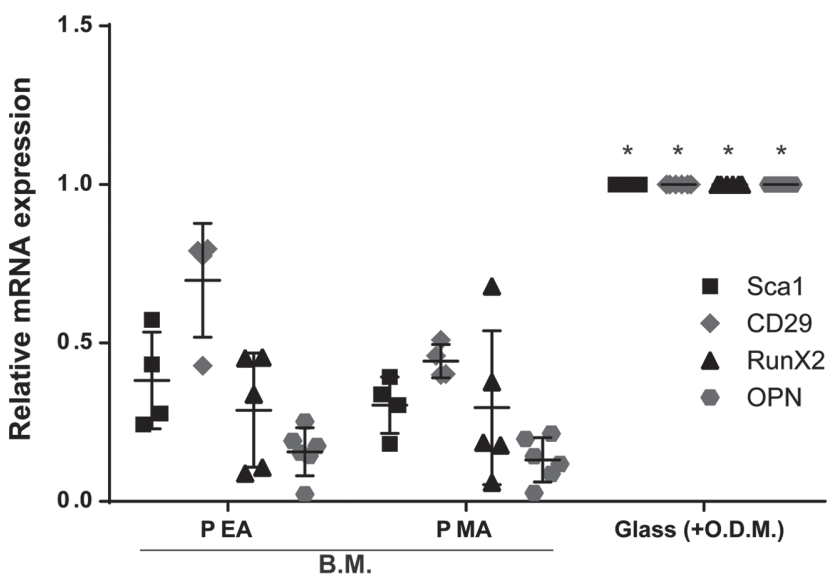

b)

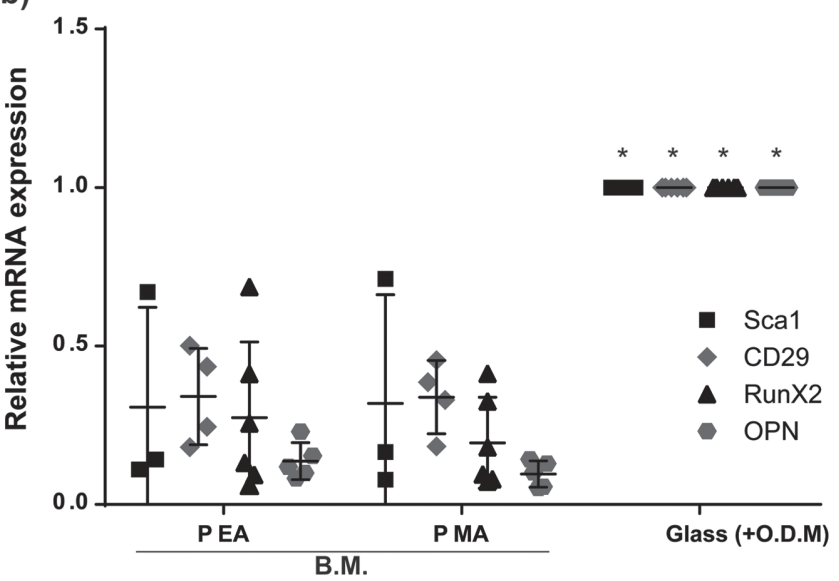

Figure 8. Analysis of relative mRNA expression after inhibition of contractility. a,b) Cells cultured under basal conditions (B.M.). Cells on control glass were induced to differentiate to osteogenic commitment (+O.D.M.). Representation of relative levels of mRNAs of genes markers of osteogenesis (Runx2 and OPN) and stemness maintenance (Scal, CD29) after $15 \mathrm{~d}$ of culture with addition of inhibitors of contractility Y-27632 and blebbistatin, respectively. Inhibition of contractility strongly reduced expression of stemness markers.

focal adhesion assembly, and cell differentiation. ${ }^{[19,41,42]}$ We have previously reported the amount of FN adsorbed on PEA and PMA to be similar at $\approx 340 \mathrm{ng} \mathrm{cm}^{-2}$ for both polymers. ${ }^{[22]}$ However, FN distribution and conformation differ significantly with interconnected FN nanonetworks on PEA whereas globular aggregates appeared on PMA (Figure 1). ${ }^{[16]}$ This work demonstrates the critical nature of this protein interface in MSC functionality. The MSCs acted with far greater growth and differentiation potential when biomimetic FN networks rather than FN with globular morphology was used as the culture substrate.

Adhesion-derived tension is critical for several processes, including cell survival ${ }^{[43]}$ and MSC differentiation. ${ }^{[13,44]}$ In this work, we first evaluated the role of FN nanonetworks in initial cell adhesion of mMSC and signaling. Focal adhesions are multiprotein associated complexes that act as a link between extracellular matrix (ECM) and actin cytoskeleton. Their structure, composition, and dynamics depend on the information received from the extracellular protein environment-in this case adsorbed FN in different conformations. In fact, it has been found a direct correlation between their size and functionality. Classically, adipogenesis (small adhesions, low intracellular tension) and osteogenesis (large adhesions, high intracellular tension) are used as exemplars. ${ }^{[5,6,45]}$ However, much less in understood about the MSC phenotype itself in terms of tension phenotype.

PEA, supporting fibrillogenesis, presented enhanced focal adhesion formation in terms of vinculin, talin, and tensin expression (Figure 2a and Figure S1, Supporting Information). Quantification of focal adhesion surface area showed significantly higher values for MSCs on the fibrillar networks although the size distribution of totality of focal adhesions remained similar for all substrates (Figure 3a,b). This suggests that the FN network assembled on PEA enhances focal adhesion number but not size.

FAK, a downstream effector of integrin ligation to the extracellular environment ${ }^{[5,6]}$ such as FN, can simultaneously activate multiple signaling pathways that regulate cell migration, survival, proliferation, and differentiation. ${ }^{[46]}$ Activation of FAK is produced after phosphorylation at Y-397, the autophosphorylation site and a binding point for Src and PI-3 Kinases. ${ }^{[4]}$ The pFAK/FAK ratio was significantly higher in PEA than on PMA or glass (Figure 3c), which correlates to the unfolding of FN during fibrillogenesis and subsequent availability of critical domains for cell adhesion such as Arg-Gly-Asp (RGD) and ProHis-Ser-Arg-Asn (PHSRN) as we, and others, have described before. ${ }^{[23,48,49]}$

These data show that MSCs on PEA are adhesion primed to increase responsiveness but do not reveal if tension itself is important in stem cell growth. Addition of contractility inhibitors, blebbistatin or Y-27632, reduced ability to commit to osteogenic differentiation (as expected) and ability to retain the MSC phenotype showing that MSC growth and maintenance of stemness require intracellular tension. This ties in with literature on human MSCs showing that while adipogenesis requires almost no intracellular tension, the MSC growth requires slightly reduced tension than fibroblastic differentiation and much lower tension than osteobalstic differentiation. ${ }^{[50,51]}$

\section{Conclusions}

This work shows, for the first time, that in order to fully potentiate the ability of biomaterials to control MSC growth and differentiation (as in the niche), full attention needs to be given to the intermediate protein layer. We hypothesize that biology has evolved to use certain protein conformations to achieve a full range of cell control. Thus, when we use a biomimetic protein conformation, we achieve a significantly more dynamic range of MSC growth and differentiation ability. Material-driven assembly of FN nanonetworks on PEA sustain stem cell growth maintaining stemness and then MSCs can be induced to differentiation on the same substrate by changing the external conditions.

\section{Experimental Section}

Materials: Polymers were synthesized by radical polymerization of methyl acrylate and ethyl acrylate (Sigma-Aldrich). The initiator was 
benzoin at 1 and $0.35 \mathrm{wt} \%$ respectively ( $98 \%$ pure, Scharlau). Afterward, the samples were dried to constant weight. Spin coating was used to coat glass coverslips and produce thin films of these polymers (Brewer Science). Polymer solutions were made in toluene with $6 \%$ PMA or $2.5 \%$ PEA and spun at $2000 \mathrm{rpm}$ for $30 \mathrm{~s}$. Finally, samples were dried at $60^{\circ} \mathrm{C}$ in vacuum. For cell culture experiments samples were sterilized by UV exposure for $30 \mathrm{~min}$

AFM: AFM was performed in a NanoScope III from Digital Instruments (Santa Barbara, CA) operating in the tapping mode in air; the Nanoscope 5.30r2 software version was used. Si-cantilevers from Veeco (Manchester, UK) were used with force constant of $2.8 \mathrm{~N} \mathrm{~m}^{-1}$ and resonance frequency of $75 \mathrm{kHz}$. The phase signal was set to zero at a frequency $5 \%-10 \%$ lower than the resonance one. Drive amplitude was $200 \mathrm{mV}$ and the amplitude setpoint Asp was 1.4 V. The ratio between the amplitude setpoint and the free amplitude Asp/A0 were kept equal to 0.7 .

Protein Adsorption: FN from human plasma (Sigma-Aldrich) was adsorbed on the different substrates by covering the material films with FN solutions of concentration $20 \mu \mathrm{g} \mathrm{mL}^{-1}$ in Dulbecco's phosphate saline buffer (DPBS). After adsorption, samples were rinsed in DPBS to eliminate the nonadsorbed protein.

Cell Culture: Murine embryonic mesenchymal stem cells (mMSCs) C3H10T1/2 (RIKEN Cell Bank, Japan) were cultured in DMEM with $10 \% \mathrm{FBS}$ and antibiotics $1 \%$ (penicillin/streptomycin) at $37{ }^{\circ} \mathrm{C}$ in a humidified atmosphere of $5 \% \mathrm{CO}_{2}$ (growth medium and basal medium composition, B.M.). For differentiation experiments, mMSCs were plated on glass covers coated with $\mathrm{FN}$, at a density of 10000 cells cm for osteogenic and basal conditions, 30000 cells $\mathrm{cm}^{-2}$ for adipogenic differentiation and cultured for $48 \mathrm{~h}$ in DMEM growth media. After cells reached $70-80 \%$ confluence, differentiation was induced with osteogenic media consisting of DMEM growth medium supplemented with ascorbic Acid $50 \mu \mathrm{g} \mathrm{mL} \mathrm{m}^{-1}$, glycerophosphate $10 \times 10^{-3} \mathrm{M}$, and dexamethasone $0.1 \times 10^{-6} \mathrm{M}$ or adipogenic media consisting of DMEM growth medium supplemented with 3-isobutyl-1-methyl-xanthine $0.5 \times 10^{-3} \mathrm{M}$, indomethacin $60 \times 10^{-6} \mathrm{M}$, and hydrocortisone $0.5 \times 10^{-6} \mathrm{M}$. Media was changed every $3 \mathrm{~d}$ until end-point assay. All differentiation experiments were finished after $15 \mathrm{~d}$.

Differentiation media were used only in differentiation control samples and differentiation induced experiments.

Immunohistochemistry Assays: After culture, cells were washed in DPBS and fixed in a $10 \%$ formalin solution (Sigma-Aldrich) at $4{ }^{\circ} \mathrm{C}$ for $30 \mathrm{~min}$. Samples were then rinsed with DPBS and maintained in a permeabilizing buffer (103 $\mathrm{g} \mathrm{L}^{-1}$ sucrose, $2.92 \mathrm{~g} \mathrm{~L}^{-1} \mathrm{NaCl}, 0.6 \mathrm{~g} \mathrm{~L}^{-1} \mathrm{MgCl}_{2}$, $4.76 \mathrm{~g} \mathrm{~L}^{-1}$ 4-(2-hydroxyethyl)-1-piperazineethanesulfonic acid (HEPES) buffer, $5 \mathrm{~mL} \mathrm{~L} \mathrm{~L}^{-1}$ Triton $\mathrm{X}-100, \mathrm{pH} 7.2$ ) at room temperature for $5 \mathrm{~min}$. Afterward, samples were incubated with primary antibody in blocking buffer $1 \%$ bovine serum albumin (BSA)/DPBS at room temperature for $1 \mathrm{~h}$; The samples were then rinsed twice in 0.5\% Tween 20/DPBS and incubated with the secondary antibody and BODIPY FL phallacidin (Invitrogen) 1:100 at room temperature for $1 \mathrm{~h}$. Finally, samples were washed twice in $0.5 \%$ Tween 20/DPBS before mounting with Vectashield containing DAPI (Vector Laboratories) and observed under an epifluorescence microscope (Nikon Eclipse 80i).

For cell adhesion studies monoclonal primary mouse antibodies diluted 1:400 against vinculin (Sigma-Aldrich), talin (Sigma-Aldrich), and tensin (abcam) and $\mathrm{Cy}^{3}$ conjugated secondary antibody (Jackson Immunoresearch) diluted 1:200 were used to detect focal adhesions. For evaluation of contractility antiphospho-myosin light chain antibody (Cell signaling) diluted 1:200 and $\mathrm{Cy}^{3}$ conjugated secondary antibody (Jackson Immunoresearch) diluted 1:200 were used. For experiments including contractility inhibitors, Y-27632 or blebbistatin were added to culture medium at different concentrations $\left(10 \times 10^{-6}\right.$ and $\left.20 \times 10^{-6} \mathrm{M}\right)$ after $2 \mathrm{~h}$ of culture.

For evaluation of differentiation, several specific markers were used. Osteogenic differentiation was assessed using Runx2 (abcam) and osteopontin (Santa cruz Bioterchnology) dilution 1:100 as primary antibodies. $\mathrm{Cy}^{3}$ (Jackson Immunoresearch) and Alexa Fluor 488 (Invitrogen) diluted 1:200 were used as secondary antibodies.
Adipogenic differentiation was detected by observation of lipid levels that were qualitatively assessed by a standard Oil red $O$ staining protocol. Briefly, cells were washed in DPBS. Immediately before use, 30 $\mathrm{mL}$ of a stock solution of Oil red O (3 $\mathrm{mg} \mathrm{mL}^{-1}$ in $99 \%$ isopropanol) was mixed with $20 \mathrm{~mL} \mathrm{diH} \mathrm{H}_{2} \mathrm{O}$, filtered, and applied for 10-15 min at room temperature to cells preequilibrated with $60 \%$ isopropanol.

Maintenance of stemness was evaluated by detection of specific mMSC marker Scal (abcam) diluted 1:200 as primary antibody and Alexa Fluor 488 antibody (Jackson Immunoresearch, diluted 1:200) as secondary antibody.

Gene Expression Analysis by Quantitative Real Time PCR: Total RNA was extracted from MC3T3 cells cultured for 3,15 or $30 \mathrm{~d}$ under different experimental conditions using RNeasy micro kit (Qiagen) according to the manufacturer's protocol. RNA quantity and integrity was measured with a NanoDrop 1000 (ThermoScientific). Then, $150 \mathrm{ng}$ of RNA were reverse transcribed using the Superscript III reverse transcriptase (Invitrogen) and oligo dT primer (Invitrogen). Real-time qPCR was carried out using the Sybr select master mix and 7500 Real Time PCR system from Applied Biosystems. The reactions were run in triplicate for both technical and biological replicas. The primers used for amplification were designed based on sequences found in the GenBank database and included: Runx2 (NM_001146038.1, Forward: 5'-TGA GAG TAG GTC TCC CGC CT-3', Reverse: 5'-TGT GGA TTA AAA GGA CTT GGT GC-3') and Osteopontin (NM 001204201.1, Forward: 5'-TTT GCC TGT TTC GCA TTG C-3', Reverse: $5^{\prime}$-TGG GTG CAG GCT GTA AAG CT-3') for osteogenic differentiation. PPAR $\gamma 2$ (NM 001127330.1, Forward: 5'-AGC AAA GAG GTC GCC ATC C-3', Reverse: $5^{\prime}$-CTT GCA CGG CTT CTA CG-3') and LPL (NM 008509.2, Forward: 5'-TGC CCT AAC GAC CCC TGA A-3', Reverse: 5'-CAC TTA GAC ACA GAG TCT GC-3') were used for adipogenic differentiation. Scal (NM 001271416.1, Forward: 5'-GAC CCT GGA GGC ACA CAG CC-3', Reverse: $5^{\prime}$-CAT GTG GGA ACA TTC CAC GAC CCC-3') and CD29 (Forward: 5'-GGA GGA ATG TAA CAC GAC TG-3', Reverse: 5'-TCC CCA CTC CTC ACT TAG GAA TC-3') were used for assessment of maintenance of stemness.

The fractional cycle number at which fluorescence passed the threshold (Ct values) was used for quantification using the comparative $\mathrm{Ct}$ method. Sample values were normalized to the threshold value of housekeeping gene GAPDH. The $\mathrm{Ct}$ value of the positive control (glass substrate) was used as a reference.

FAK and PFAK Detection and Quantification: For FAK assays cells were cultured under serum-free conditions in order to reduce background signal. Cells were then seeded on FN-coated materials in serum-free medium. After $3 \mathrm{~h}$ of culture, cells were lysed with radioimmunoprecipitation assay (RIPA) buffer (Tris- $\mathrm{HCl} 50 \times$ $10^{-3} \mathrm{M}, 1 \%$ Nonidet P-40, $0.25 \% \mathrm{Na}$ deoxycholate, $\mathrm{NaCl} 150 \times 10^{-3} \mathrm{M}$, ethylenediaminotetraacetic acid (EDTA) $1 \times 10^{-3} \mathrm{M}$ ) containing protease inhibitor cocktail tablets (Roche). Proteins were concentrated using Microcon YM-30 Centrifugal Filter devices (Millipore) as the manufacturer described. To determine FAK protein expression and its phosphorylated form (pFAK), concentrated samples were subjected to $7 \%$ sodium dodecyl sulfate polyacrylamide gel electrophoresis (SDS PAGE). Proteins were transferred to a positively charged polyvinylidene fluoride (PVDF) membrane (GE Healthcare) using a semidry transfer cell system (Biorad) and blocked by immersion in $5 \%$ semiskimmed milk in phosphate bufered saline (PBS) for $30 \mathrm{~min}$ at room temperature. The blot was incubated with anti-FAK (abcam, $400 \mathrm{ng} \mathrm{mL}^{-1}$ ) and antipFAK (abcam, $1 \mathrm{mg} \mathrm{mL}^{-1}$ ) in PBS containing $0.1 \%$ Tween 20 and $2 \%$ semiskimmed milk. After several washes with PBS/0.1\% Tween 20 , the blot was incubated in horseradish peroxidase-conjugated antibody (GE Healthcare) diluted 1:25 000 for FAK and 1:10 000 for pFAK in PBS containing $0.1 \%$ Tween 20 and $2 \%$ semiskimmed milk for $1 \mathrm{~h}$ at room temperature. Supersignal West Femto Maximum Sensitivity Substrate (Pierce) was used before X-ray exposition.

Image Analysis: To analyze focal adhesions, vinculin images were segmented by Image), using Trainable Weka Segmentation plug in to create a binary mask. After segmentation, focal adhesion size and number were determined using different commands of the same software. Values of focal adhesion size frequency were represented using 
GraphPad Prism 6.0. using a bin width of $0.2 \mu \mathrm{m}$. Cell morphology was analyzed by calculation of different parameters using Image) software. Cell spreading area and roundness $\left(4 \times\right.$ area $\pi^{-1} \times$ [major axis] $\left.{ }^{2}\right)$ were calculated by evaluation of at least 40 cells for each condition. Western blot bands pixel density and staining intensity of immunofluorescence images were quantified using Image) software.

Statistical Analysis: All experiments were performed at least three times, unless otherwise noted. Data are reported as mean \pm standard deviation. Results were analyzed by one-way analysis of variance (ANOVA) using SYSTAT 8.0 (SPSS). If treatment level differences were determined to be significant, pairwise comparisons were performed using a Tukey post hoc test. A 95\% confidence level was considered to be significant.

\section{Supporting Information}

Supporting Information is available from the Wiley Online Library or from the author. All original data related to this manuscript is within the depository of the University of Glasgow with DOI:10.5525/gla. researchdata.338.

\section{Acknowledgements}

AFM was performed under the technical guidance of the Microscopy Service at the Universidad Politécnica de Valencia, whose advice is greatly appreciated. The authors appreciate help provided by A. Rodrigo-Navarro for design of the cover. The financial support from Medical Research Council (MRC) (MR/L022710/1), European Research Council (ERC) (HealInSynergy, 306990), the Spanish Ministry of Economy and Competitiveness (MINECO) (through the MAT201569315-C3-1-R), and Fondo Europeo de Desarrollo Regional (FEDER) funds project are acknowledged. CIBER-BBN is an initiative funded by the VI National R\&D\&I Plan 2008-2011, Iniciativa Ingenio 2010, Consolider Program, CIBER Actions and financed by the Instituto de Salud Carlos III with assistance from the European Regional Development Fund.

Received: May 11, 2016 Revised: July 1, 2016 Published online: August 4, 2016

[1] S. Harada, G. A. Rodan, Nature 2003, 423, 349.

[2] C. A. Gregory, J. Ylostalo, D. J. Prockop, Sci. STKE 2005, 294, pe37.

[3] W. Wagner, P. Horn, M. Castoldi, A. Diehlmann, S. Bork, R. Saffrich, V. Benes, J. Blake, S. Pfister, V. Eckstein, A. D. Ho, PLoS One 2008, 3, e2213.

[4] R. Izadpanah, D. Kaushal, C. Kriedt, F. Tsien, B. Patel, J. Dufour, B. A. Bunnell, Cancer Res. 2008, 68, 4229.

[5] R. McBeath, D. M. Pirone, C. M. Nelson, K. Bhadriraju, C. S. Chen, Dev. Cell 2004, 6, 483.

[6] K. A. Kilian, B. Bugarijab, B. T. Lahnb, M. Mrksicha, Proc. Natl. Acad. Sci. USA 2010, 107, 4872

[7] C. Yang, M. W. Tibbitt, L. Basta, K. S. Anseth, Nat. Mater. 2014, 13, 645.

[8] J. M. Curran, R. Chen, J. A. Hunt, Biomaterials 2006, 27, 4783.

[9] D. S. W. Benoit, M. P. Schwartz, A. R. Durney, K. S. Anseth, Nat. Mater. 2008, 7, 816.

[10] M. J. Dalby, N. Gadegaard, A. S. G. Curtis, R. O. C. Oreffo, Curr. Stem Cell Res. Ther. 2007, 2, 129.
[11] S. Oha, K. S. Brammera, Y. S. J. Lib, D. Tengb, A. J. Engler, S. Chien, S. Jin, Proc. Natl. Acad. Sci. USA 2009, 106, 2130.

[12] R. J. McMurray, N. Gadegaard, P. M. Tsimbouri, K. V. Burgess, L. E. McNamara, R. Tare, K. Murawski, E. Kingham, R. O. C. Oreffo, M. J. Dalby, Nat. Mater. 2011, 10, 637.

[13] A. J. Engler, S. Sen, H. L. Sweeney, D. E. Discher, Cell 2006, 126, 677.

[14] P. M. Gilbert, K. L. Havenstrite, K. E. G. Magnusson, A. Sacco, N. A. Leonardi, P. Kraft, N. K. Nguyen, S. Thrun, M. P. Lutolf, H. M. Blau, Science 2010, 329, 1078.

[15] O. Chaudhuri, L. Gu, D. Klumpers, M. Darnell, S. A. Bencherif, J. C. Weaver, N. Huebsch, H. Lee, E. Lippens, G. N. Duda, D. J. Mooney, Nat. Mater. 2015, 15, 326.

[16] M. Salmerón-Sánchez, P. Rico, D. Moratal, T. T. Lee, J. E. Schwarzbauer, A. J. García, Biomaterials 2011, 32, 2099.

[17] J. Ballester-Beltrán, M. Cantini, M. Lebourg, P. Rico, D. Moratal, A. J. García, M. Salmerón-Sánchez, J. Mater. Sci. Mater. Med. 2012, 23, 195.

[18] B. G. Keselowsky, D. M. Collard, A. J. García, Biomaterials 2004, 25, 5947.

[19] B. G. Keselowsky, D. M. Collard, A. J. García, Proc. Natl. Acad. Sci. USA 2005, 102, 5953.

[20] K. E. Michael, D. W. Dumbauld, K. L. Burns, S. K. Hanks, A. J. García, Mol. Biol. Cell 2009, 20, 2508.

[21] Y. Mei, K. Saha, S. R. Bogatyrev, J. Yang, A. L. Hook, Z. I. Kalcioglu, S. Cho, M. Mitalipova, N. Pyzocha, F. Rojas, K. J. Van Vliet, M. C. Davies, M. R. Alexander, R. Langer, R. Jaenisch, D. Anderson, Nat. Mater. 2010, 9, 768.

[22] P. Rico, J. C. Rodríguez-Hernández, D. Moratal, G. Altankov, M. Monleón Pradas, M. Salmerón-Sánchez, Tissue Eng., Part A 2009, 15, 3271.

[23] P. Rico, C. González-García, T. A. Petrie, A. J. García, M. SalmerónSánchez, Colloids Surf., B 2010, 78, 310.

[24] N. Brizuela Guerra, C. González-García, V. Llopis, J. C. RodríguezHernández, D. Moratal, P. Rico, M. Salmerón-Sánchez, Soft Matter 2010, 6, 4748.

[25] C. A. Reznikoff, D. W. Brankow, C. Heidelberger, Cancer Res. 1973, 33, 3231.

[26] D. F. Pinney, C. P. Emerson, Environ. Health Perspect. 1989, 80, 221.

[27] C. González-García, D. Moratal, R. O. C. Oreffo, M. J. Dalby, M. Salmerón-Sánchez, Integr. Biol. 2012, 4, 531.

[28] M. F. Pittenger, A. M. Mackay, S. C. Beck, R. K. Jaiswal, R. Douglas, J. D. Mosca, M. A. Moorman, D. W. Simonetti, S. Craig, D. R. Marshak, Science 1999, 284, 143

[29] M. Dominici, I. Mueller, I. Slaper-Cortenbach, F. Marini, D. Krause, R. Deans, A. Keating, D. J. Prockop, E. Horwitz, Cytotherapy 2006, 8,315 .

[30] G. Chamberlain, J. Fox, B. Ashton, J. Middleton, Stem Cells 2007, 25, 2739.

[31] G. R. Kirkham, S. H. Cartmell, Top. Tissue Eng. 2007, 3, 1

[32] R. J. Kim, S. Kim, K. Roh, S. Park, J. Park, K. Kang, G. Kong, B. Tang, Y. Yang, E. A. Kohn, L. M. Wakefield, J. Nam, Cancer Lett. 2010, 287, 172.

[33] A. Satelli, S. Li, Cell. Mol. Life Sci. 2011, 68, 3033.

[34] M. Griffin, S. Sen, H. L. Sweeney, D. E. Discher, J. Cell Sci. 2004, 117,5855

[35] P. Roca-Cusachs, T. Iskratsch, M. P. Sheetz, J. Cell Sci. 2012, 125, 3025.

[36] K. Kaibuchi, S. Kuroda, M. Amano, Annu. Rev. Biochem. 1999, 68, 459.

[37] S. Narumiya, T. Ishizaki, M. Ufhata, Methods Enzymol. 2000, 325, 273.

[38] M. Kovacs, J. Tóth, C. Hetényi, A. Málnási-Csizmadia, J. Sellers, J. Biol. Chem. 2004, 279, 35557 
[39] M. J. Dalby, N. Gadegaard, R. Tare, A. Andar, M. O. Riehle, P. Herzyk, C. D. W. Wilkinson, R. O. C. Oreffo, Nat. Mater. 2013, 6, 997.

[40] J. E. Phillips, T. A. Petrie, F. P. Creighton, A. J. Garcia, Acta Biomater. 2010, 6, 12.

[41] B. G. Keselowsky, D. M. Collard, A. J. García, J. Biomed. Mater. Res. A 2003, 66, 247

[42] B. G. Keselowsky, D. M. Collard, A. J. García, Biomaterials 2004, 25, 5947.

[43] C. S. Chen, M. Mrksich, S. Huang, G. M. Whitesides, D. E. Ingber, Science 1997, 276, 1425.

[44] D. E. Discher, D. J. Mooney, P. W. Zandstra, Science 2009, 324, 1673.

[45] B. Geiger, A. Bershadsky, R. Pankov, K. M. Yamada, B. G. Correspondence, Nat. Rev. Mol. Cell Biol. 2001, 2, 793.
[46] S. K. Mitra, D. A. Hanson, D. D. Schlaepfer, Nat. Rev. Mol. Cell Biol 2005, 6, 56.

[47] M. D. Schaller, J. D. Hilderbrand, J. D. Shannon, J. W. Fox R. R. Vines, T. Parsons, Mol. Cell. Biol. 1994, 14, 1680.

[48] S. T. Lee, J. I. Yun, Y. S. Jo, M. Mochizuki, A. J. van der Vlies, S. Kontos, J. E. Ihm, J. M. Lim, J. A. Hubbell, Biomaterials 2010, 31, 1219.

[49] F. A. Vanterpool, M. Cantini, F. P. Seib, M. Salmerón-Sánchez, Bio Res. Open Access 2014, 3, 286.

[50] M. J. Dalby, N. Gadegaard, R. O. C. Oreffo, Nat. Mater. 2014, 13 558.

[51] P. M. Tsimbouri, R. J. McMurray, K. V. Burgess, E. V. Alakpa, P. M. Reynolds, K. Murawski, E. Kingham, R. O. C. Oreffo, N. Gadegaard, M. J. Dalby, ACS Nano 2012, 6, 10239. 